\title{
Therapeutic Approach to the Management of Pediatric Demyelinating Disease: Multiple Sclerosis and Acute Disseminated Encephalomyelitis
}

\author{
J. Nicholas Brenton ${ }^{1}$ (D) $\cdot$ Brenda L. Banwell ${ }^{2}$
}

Published online: 23 October 2015

(C) The American Society for Experimental NeuroTherapeutics, Inc. 2015

\begin{abstract}
Acquired pediatric demyelinating diseases manifest acutely with optic neuritis, transverse myelitis, acute disseminated encephalomyelitis, or with various other acute deficits in focal or polyfocal areas of the central nervous system. Patients may experience a monophasic illness (as in the case of acute disseminated encephalomyelitis) or one that may manifest as a chronic, relapsing disease [e.g., multiple sclerosis (MS)]. The diagnosis of pediatric MS and other demyelinating disorders of childhood has been facilitated by consensus statements regarding diagnostic definitions. Treatment of pediatric MS has been modeled after data obtained from clinical trials in adult-onset MS. There are now an increasing number of new therapeutic agents for MS, and many will be formally studied for use in pediatric patients. There are important efficacy and safety concerns regarding the use of these therapies in children and young adults. This review will discuss acute management as well as chronic immunotherapies in acquired pediatric demyelination.
\end{abstract}

Keywords Pediatric multiple sclerosis $\cdot$ Acute disseminated encephalomyelitis · Treatment $\cdot$ Acquired demyelinating syndrome

J. Nicholas Brenton jnb8h@virginia.edu

1 Department of Neurology, Division of Pediatric Neurology, University of Virginia, PO Box 800394, Charlottesville, VA 22908, USA

2 Division of Neurology, Children's Hospital of Philadelphia, 3501 Civic Center Boulevard, Colket Translational Research Building, 10th floor, Philadelphia, PA 19104, USA

\section{Introduction}

A first demyelinating attack in childhood or adolescence is known as an acquired demyelinating syndrome (ADS). The course of a first demyelinating attack may be monophasic or may represent the sentinel attack of an underlying, chronic demyelinating disorder such as multiple sclerosis (MS) or neuromyelitis optica (NMO). Pediatric ADS occurs with a reported incidence of $0.5-1.66$ per 100,000 children [1-4], and may present as optic neuritis, transverse myelitis, acute disseminated encephalomyelitis (ADEM), or as various other monofocal or polyfocal deficits. In pediatric ADS cohorts, optic neuritis constitutes $22-36 \%$, transverse myelitis 3$22 \%$, and ADEM 19-32\% of cases. Other monofocal or polyfocal presentations (21-46\%) comprise the remainder of ADS cases [1-3,5].

The reported proportion of children with ADS that are ultimately diagnosed with MS is variable and ranges from $15 \%$ to $45 \%$ [2, 3, 6-9]. Up to $10.5 \%$ of all patients with MS experience the clinical onset of MS before the age of 18 years [10]. Though the pathobiology appears similar to that of adultonset MS, MS in childhood manifests almost exclusively as a relapsing-remitting MS (RRMS) phenotype. When compared with patients with adult-onset MS with similar disease duration, pediatric patients experience a relapse rate 2-3 times higher than that of adults in the first few years of disease $[11,12]$. The mechanisms underlying higher relapse rates may conceptually relate to greater immune activation, to differences in the balance of effector and regulatory immune cells, to age-related differences in immune cell access into the central nervous system (CNS), or to intracerebral factors that influence the formation of lesions. Comprehensive, comparative biological studies are required to better understand the impact of age on clinical disease expression. The more inflammatory initial course of pediatric-onset MS highlights 
the rationale for prompt initiation of immunomodulatory therapies in children.

There are now 12 Food and Drug Administration (FDA)approved medications for the treatment of RRMS, with 1 new injectable therapy, 3 oral medications, and 1 infusion therapy approved in the last 5 years. With aims of greater disease suppression comes a higher risk of adverse, and potentially fatal, side effects. Though safety and efficacy data within adult cohorts have been demonstrated, the effects and safety profiles of these new therapies have yet to be determined in pediatric patients.

In this review, we will focus on current diagnostic tools available for differentiating and diagnosing the spectrum of ADS of childhood, with specific attention to that of ADEM and MS. We will review data on the acute care of a demyelinating attack in addition to current and upcoming therapies for MS. Particular attention will be paid to upcoming clinical trials in pediatric MS and the unique obstacles we face as these trials are conceptualized and commenced.

\section{Making a Diagnosis: History, Examination, Laboratory, and Radiologic Evaluation}

At initial presentation, there is inherent difficulty in distinguishing monophasic, self-limited ADS from those that will go on to manifest with a chronic neuroinflammatory condition. The International Pediatric MS Study Group (IPMSSG) has proposed consensus guidelines to assist in the diagnosis of the major neuroinflammatory diseases of childhood and supply a common terminology for all providers [13].

\section{ADEM}

Polyfocal ADS can manifest with or without encephalopathy. If the patient presents without encephalopathy, a diagnosis of polyfocal clinically isolated syndrome is conferred and the patient is subsequently considered at high risk for going on to meet diagnostic criteria for MS. Children with encephalopathy (mental status changes or behavioral alterations) and concurrent multifocal demyelination, manifesting with polysymptomatic neurologic symptoms, meet diagnostic criteria for ADEM [13]. The differential diagnoses include CNS infection, mitochondrial disease, antibody-associated encephalopathies, and metabolic syndromes.

Patients with ADEM tend to be prepubertal, with $80 \%$ of childhood cases occurring in those aged 10 years or younger. ADEM has a peak incidence at 5-8 years [14-18]. There is a seasonal predilection for fall-to-winter occurrence, and there is often a history of preceding infection or vaccination; however, up to a quarter of cases may lack a clear history of either $[17,19]$. There are no current serum or cerebrospinal fluid biomarkers for ADEM; however, cerebrospinal fluid often demonstrates a mild-to-moderate pleocytosis and elevated protein. Oligoclonal bands may be transiently seen in up to $10 \%$ of patients $[15,20]$. Autoantibodies, including antimyelin oligodendrocyte glycoprotein and anti-aquaporin-4, have been reported in children who meet criteria for ADEM, though the exact implications of these antibodies have yet to be fully elucidated $[21,22]$. The presence of anti-aquaporin-4 antibodies should prompt consideration of NMO, as the presence of the antibody strongly predicts future relapse.

Typical magnetic resonance imaging (MRI) changes noted in ADEM include multifocal fluid attenuation inversion recovery (FLAIR) and T2-hyperintense lesions that predominately involve the white matter of the brain and spinal cord. Lesions are often large $(>1-2 \mathrm{~cm})$ with poorly defined borders. Deep gray matter involvement is often observed. Imaging abnormalities frequently resolve as the patient sustains clinical recovery $[8,15,17,18]$. Monophasic ADEM, at times, may be difficult to distinguish from the first attack of MS; however, MRI findings of diffuse, bilateral T2-hyperintense lesions in addition to absence of T1-hypointense "black holes" and lack of periventricular lesions weigh heavily in favor of a diagnosis of ADEM [23, 24].

ADEM is most often a monophasic illness and the vast majority of patients make a complete recovery; however, a small subset (6-29\%) of children with an initial diagnosis of ADEM will have future demyelinating attacks characteristic of MS $[6,25]$. A small percentage $(\leq 10 \%)$ of patients with ADEM will experience a biphasic course, with a subsequent second attack of ADEM-termed multiphasic ADEM $[15,26]$. Rarely, ADEM may be followed by monophasic or recurrent optic neuritis and may also precede or follow a diagnosis of anti-N-methyl-D-aspartate (NMDA) receptor encephalitis [27, 28].

\section{MS}

MS is characterized by multifocal areas of demyelination within the CNS and by accrual of these lesions over time. By recent diagnostic criteria, MS may be diagnosed at the time of a first clinical attack, as long as imaging at baseline scan demonstrates evidence of "dissemination in time and space" (Fig. 1) [29]. Dissemination in space (DIS) is evidenced by the presence of at least one T2-hyperintense lesion in at least 2 of 4 CNS areas: periventricular, juxtacortical, infratentorial, or spinal cord. Dissemination in time (DIT) is demonstrated at baseline not only by the presence of a clinically silent, enhancing lesion, as well as nonenhancing lesion(s), but may also be confirmed by the appearance of a new T2 or gadoliniumenhancing lesion on follow-up MRI.

Importantly, the use of the $2010 \mathrm{McDonald}$ criteria for MS, when applied at the time of a first attack and single MRI scan, have similar negative and positive predictive values when 
Fig. 1 Algorithm for diagnosis of multiple sclerosis (MS) in a pediatric patient. $\mathrm{ADEM}=$ acute disseminated encephalomyelitis; MRI = magnetic resonance imaging DIS = dissemination in space with presence of at least one, T2-hyperintense lesion in at least 2 of the following 4 central nervous system areas: periventricular, juxtacortical, infratentorial, or spinal cord; DIT $=$ dissemination in time demonstrated by new $\mathrm{T} 2$ or gadolinium-enhancing lesions on a follow-up MRI compared with a baseline scan or simultaneous asymptomatic gadoliniumenhancing and nonenhancing lesions on a single scan $[13,29]$

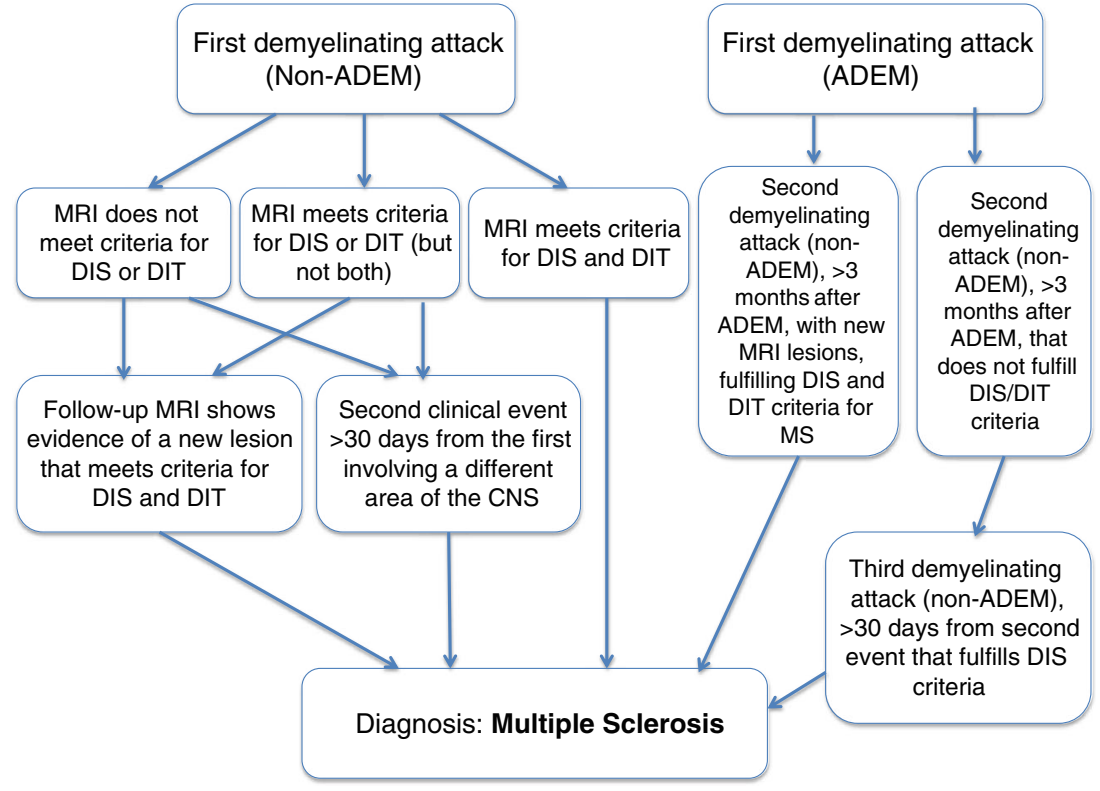

applied to youth as they do for patients with adult-onset MS. However, application of the 2010 criteria for DIS and DIT at onset in children younger than 11 years of age has a much lower positive predictive value, and thus should be utilized with caution in this context [30]. It is advisable in young children to confirm DIT by serial imaging if diagnostic certainty is in question. Furthermore, the 2010 criteria do not apply in the context of an ADEM presentation (as the high volume of T2 lesions often involves the areas required to fulfill DIS criteria, and variable enhancement of regions that may be considered "asymptomatic" could fulfill DIT criteria).

Figure 1 outlines the means of confirming a diagnosis of MS in pediatric patients with an ADEM-like first attack. Briefly, a diagnosis of MS may be conferred if the child has $\geq 2$ non-ADEM attacks involving different CNS areas separated by at least 30 days or 1 non-ADEM attack $>90$ days from initial ADEM along with MRI features that meet McDonald 2010 dissemination in time and space criteria [13]. The 90-day consideration was agreed upon by consensus based upon clinical experience in caring for children with ADEM, in whom clinical symptoms of a single event can wax and wane over a period of $>30$ days.

The likelihood that ADS represents the first attack of MS can be stratified by initial presentation, age of disease onset, and presence of findings on brain MRI. MS risk is influenced by sex, genetic predisposition, and environment. Female sex and an age of disease onset greater than 10 years old at initial ADS are associated with a higher likelihood of MS [6, 25]. An abnormal brain MRI with clinically silent T2-hyperintense and T1-hypointense lesions at the time of first ADS along with the presence of intrathecal synthesis of oligoclonal bands are also associated with a high likelihood of MS [6, 24]. The presence of encephalopathy or a normal brain MRI at the time of ADS (i.e., optic neuritis or transverse myelitis without demyelinating lesions within the brain parenchyma) portends a lower risk of MS $[6,25,26]$. Additionally, the presence of the HLA-DRB1*1501 allele, remote Epstein-Barr virus infection, and low serum vitamin $\mathrm{D}$ appear to be predisposing factors for MS [6].

As mentioned above, pediatric patients with MS tend to have a more inflammatory course within the first 2 years of onset [11], manifesting with more frequent clinical relapses and a higher brain T2- and T1-weighted lesion volume [31-34]. Patients with pediatric-onset MS generally maintain good recovery from relapses with minimal-to-no progression in disability within the first 10 years of disease onset; however, irreversible disability and secondary progression ultimately occur at a much earlier age than in adult-onset MS [35]. Despite the lack of early measurable physical disability, cognitive impairment can be noted in up to one-third of pediatric patients with MS [36-38]. Impairments in information processing speed, verbal memory, verbal fluency, and receptive language are seen with resultant negative effects on the patient's scholarship and daily life activities [39]. Longitudinal data are required to determine patterns of cognitive loss or improvement over time, the impact of treatment on cognitive function, and the impact of early-onset MS on academic and vocational achievement into adulthood.

\section{Acute Management of Demyelinating Attacks}

Mild symptoms, not impairing daily function, may be sufficiently managed with reassurance, rehabilitation, and ongoing monitoring. For most presentations, intravenous (IV) corticosteroids are considered first-line treatment [40]. Corticosteroids have 
been shown to accelerate the speed of recovery in addition to reducing the number of active, gadolinium-enhancing lesions on MRI within a few days post-treatment [41-44]. The putative mechanism of action includes modification of cytokine responses; reduction in T-cell activation; reduction in blood-brain barrier permeability that, in turn, limits extravasation of immune cells into the CNS; and facilitating apoptosis of activated immune cells [45]. International consensus favors doses of 20 $30 \mathrm{mg} / \mathrm{kg}$ (up to $1 \mathrm{~g} /$ day) of IV methylprednisolone daily for 3-5 days. Oral prednisone, starting at $1 \mathrm{mg} / \mathrm{kg} / \mathrm{day}$ and tapered over 1-4 weeks, is considered for patients with incomplete resolution of symptoms after IV treatment. There is evidence that high-dose oral corticosteroids (1250 mg of prednisone for adults) may be as beneficial as the IV form for treating acute inflammatory, demyelinating attacks of the CNS [46-48]. In children, oral steroids are less commonly used for treating an acute attack as appropriate dosing is unclear.

In cases where steroid therapy is contraindicated or the patient fails to respond adequately to appropriately dosed IV corticosteroids, intravenous immunoglobulin (IVIg) or plasma exchange (PLEX) have been utilized. The benefit of IVIg in acute demyelinating attacks is limited to case reports and small case series [49-53], and is thought to provide benefit by directly affecting cytokine production and T-cell proliferation and by binding potential autoantibodies targeted against myelin [54, 55]. IVIg is given at a dose of $2 \mathrm{~g} / \mathrm{kg}$ divided over 2-5 days. Side effects include headache, myalgia, fever, and, rarely, aseptic meningitis. Premedication with acetaminophen and diphenhydramine may help reduce infusion-based side effects. There is potential for a severe allergic reaction with IVIg in patients with IgA deficiency; thus, obtaining a serum IgA level prior to initiating treatment is advised. Thromboembolism is another rare side effect associated with IVIg therapy.

PLEX has been used increasingly for treatment of patients with severe or life-threatening demyelination, such as patients with myelitis or brainstem involvement. PLEX is an invasive therapy. Side effects include infection (typically related to the need for an indwelling catheter), alteration of electrolyte profiles, and depletion of coagulation factors. The benefit of PLEX is likely secondary to its therapeutic removal of circulating autoantibodies and immune complexes from the blood. Typical PLEX therapy is 5-7 exchanges over the course of $10-14$ days. The benefit of PLEX is likely greatest in a primarily antibody-driven pathology (such as NMO); yet, benefits have been seen in all types of inflammatory disorders, including MS [56, 57].

\section{Comprehensive Management of Pediatric-onset MS}

A multidisciplinary team, consisting of neurology, neuropsychology, social work, and physical and occupational therapy, is essential for the care of every pediatric patient with MS. The management of known environmental factors that affect disease course is important. Studies in children have found an association of low serum vitamin $\mathrm{D}$ with a heightened risk of developing MS [6], and an increase in relapse rates in patients with confirmed MS [58]. The optimal serum concentration for 25-hydroxyvitamin D remains unknown; however, oral doses of 1000-4000 IU daily in a child to achieve a serum concentration of $30-80 \mathrm{ng} / \mathrm{ml}$ is likely appropriate. Second-hand smoke, as a result of parental smoking, appears to increase the risk of developing MS, as does the duration of exposure [59]. In addition, adolescent obesity appears to be associated with an increased risk of subsequent adult-onset MS [60, 61], though the impact of obesity upon established disease is unclear. Thus, family counseling on the importance of diet and lifestyle changes along with cessation of smoking is always indicated.

Currently, there are varied views on the concept of "adequate" disease control in MS. Clinical relapse, reduction in new, T2-bright or gadolinium-enhancing lesions on MRI, and sustained increases in the Expanded Disability Status Scale score are frequently used markers of treatment efficacy. Given the low likelihood of sustained increases in Expanded Disability Status Scale in pediatric MS, only clinical and MRI data were incorporated into the IPMSSG consensus statement, which proposed that an inadequate treatment response in a compliant pediatric patient on full-dose therapy for 6 months be defined as: 1) an increase or lack of reduction in annualized relapse rates (ARR) or new T2 or gadolinium-enhancing lesions on MRI when compared with the pretreatment period; or 2) $\geq 2$ confirmed relapses (as evidenced by clinical or MRI) within a 12-month period or less [40]. As the arsenal of more potent therapies increases, the aims of treatment have shifted towards the potential goal of "no evidence of disease activity" - a metric defined by elimination of clinical relapses, the absence of new/enlarging T2- or gadolinium-enhancing lesions, and lack of increasing sustained disability [62]. The use of "no evidence of disease activity" as a marker of treatment adequacy in children, though provocative and desirable, is a concept that continues to evolve as the treatment landscape of pediatric MS advances.

\section{First-Line Therapies for Pediatric MS}

Standard, accepted immunomodulatory therapy in MS, in the form of injections (either subcutaneous or intramuscular), has been commercially available for $>20$ years. Over time, these therapies have proven to be safe and well-tolerated (Table 1). Additionally, their therapeutic benefits have been shown in multiple adult studies to reduce relapse frequency and decrease accrual of new lesions on MRI. Important aspects of care in using these injectables include patient education on 


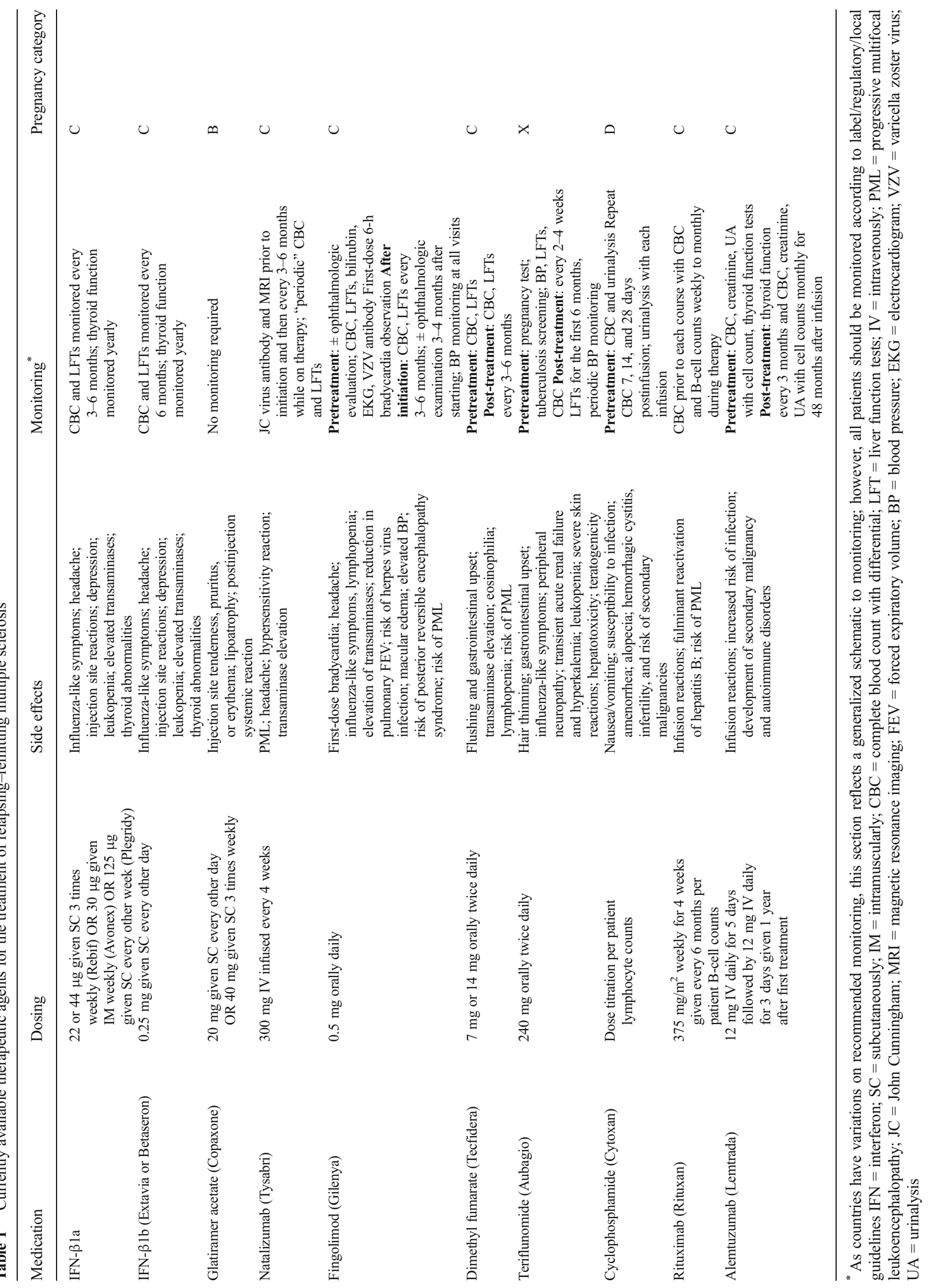


administration of the therapy in addition to the general care of injection site reactions should they occur.

It is recognized that adherence to first-line therapy in young patients with MS is not optimal $[63,64]$. This, in part, likely relates to the age-appropriate need for autonomy in addition to the social stigma of being diagnosed with a chronic disease at a time where "social camouflage" is a strong desire. For these reasons, it is important to give pediatric patients with MS a sense of control and normalcy by encouraging adherence to therapy but not allowing the therapy to significantly limit their need for independence. The authors' practice is to encourage travel and engagement in collegiate activities, despite the fact that such activities, at times, come at the cost of a single missed dose of therapy.

Retrospective or open-label studies have informed on the safety and efficacy of the current, first-line injectable therapies in children, specifically glatiramer acetate and the interferons (IFNs) (Fig. 2) [40]. At this time, there are no studies available assessing the safety and efficacy of the new oral MS drugs; however, international clinical trials are currently underway.

\section{Glatiramer Acetate}

Originally developed to simulate myelin basic protein properties, glatiramer acetate is composed of a mixture of synthetic polypeptides derived from each of 4 amino acids: L-alanine, L-glutamic acid, L-lysine, and L-tyrosine [65]. The mechanism of action is not clear but evidence suggests that it preferentially induces differentiation of CD4+ T cells into T helper 2 cells, thus promoting an anti-inflammatory state. In addition, glatiramer acetate may interact with cytokine-secreting cells prompting a shift from a proinflammatory to a more antiinflammatory cytokine profile $[66,67]$.

Glatiramer acetate is given subcutaneously at $20 \mathrm{mg}$ daily or $40 \mathrm{mg}$ thrice weekly. Patients should be educated on common adverse events, including injection site reactions. A transient systemic reaction, occurring within minutes postinjection, is typically associated with shortness of breath, chest pressure, anxiety, and flushing, and can last up to $30 \mathrm{mins}$. This phenomenon, which often occurs once but can recur with subsequent injections, has been reported in 7-14\% of pediatric patients. Several retrospective studies in pediatric MS have shown this therapy to be well tolerated, with reductions in ARR similar to that reported in adult trials [68-70]. Glatiramer acetate is perhaps the most favored diseasemodifying therapy in young women attempting pregnancy, with no known adverse events reported on the fetus [71].

\section{IFN- $\beta$}

The IFNs are a group of disease-modifying therapies that likely work via several mechanisms including shifting the cytokine balance to a more anti-inflammatory profile and reducing the trafficking of inflammatory cells across the blood-brain barrier [72]. There are 2 subclasses of IFN- $\beta-$ IFN- $\beta 1 \mathrm{a}$ and IFN- $\beta 1$ b. IFN- $\beta 1$ a can be given subcutaneously 3 times weekly at a dose of 22 or $44 \mu \mathrm{g}$ or intramuscularly at a dose of $30 \mu \mathrm{g}$ weekly. A pegylated version of IFN- $\beta 1 \mathrm{a}$ is now available and is given at a dose of $125 \mu \mathrm{g}$ every other week. IFN- $\beta 1 \mathrm{~b}$ is administered subcutaneously at $0.25 \mathrm{mg}$ every other day.

Multiple retrospective studies have demonstrated the safety and efficacy of the IFNs in pediatric MS [68, 69, 73-77]. Potential side effects include influenza-like symptoms (noted in up to $65 \%$ of patients), injection site reactions, elevated transaminases, decreased leukocytes, worsening depression, and headaches. Influenza-like symptoms can often be mitigated with nonsteroidal premedication. Expert opinion suggests that IFNs are better tolerated if they are initiated at $25-50 \%$ of the target dose followed by gradual escalation to full dose over 1-3 months [78]. Fetal exposure to IFN- $\beta$ in utero may be associated with preterm birth and a lower mean birth weight and length [71].

\section{Second-line Therapies for Pediatric MS}

When first-line therapies fail to treat a pediatric patient with MS effectively, second-line therapies are often employed. "Failure" of first-line therapies is often secondary to breakthrough disease, poor tolerance/adherence to the therapy, or a combination of both. An estimated $44 \%$ of pediatric patients experience treatment failure with a single first-line therapy. Nearly $80 \%$ of these patients are given a second injectable with a different mechanism of action. The remaining $20 \%$ are offered second-line therapies [79].

The decision to escalate treatment to second-line therapies is not straightforward and remains dependent on several factors, including severity of relapse and degree of recovery from a relapse, safety and adverse event profile of a given treatment option, and the child and family's goals and expectations (Fig. 2). There are currently a handful of therapies utilized for refractory pediatric MS (Table 1). Selection should consider the mechanism of action of a given therapy, the treatment duration required to reach maximal therapeutic benefit, the sustainability of the therapeutic strategy, and the potential effect of the therapy on a young, developing patient's neuroimmunologic system.

\section{Infusion Therapies}

\section{Natalizumab}

Natalizumab is a monoclonal antibody that is directed against the $\alpha-4$ subunit of very late activating antigen-4 - a cell surface adhesion molecule found on the majority of leukocytes. 
Fig. 2 Proposed algorithm for the approach to treating a pediatric patient with multiple sclerosis (MS) [40].

$\mathrm{GA}=$ glatiramer acetate;

$\mathrm{IFN}=$ interferon; $\mathrm{MRI}=$ magnetic resonance imaging;

AST $=$ aspartate

aminotransferase; $\mathrm{ALT}=$ alanine

transaminase; $\mathrm{WBC}=$ white

blood cells

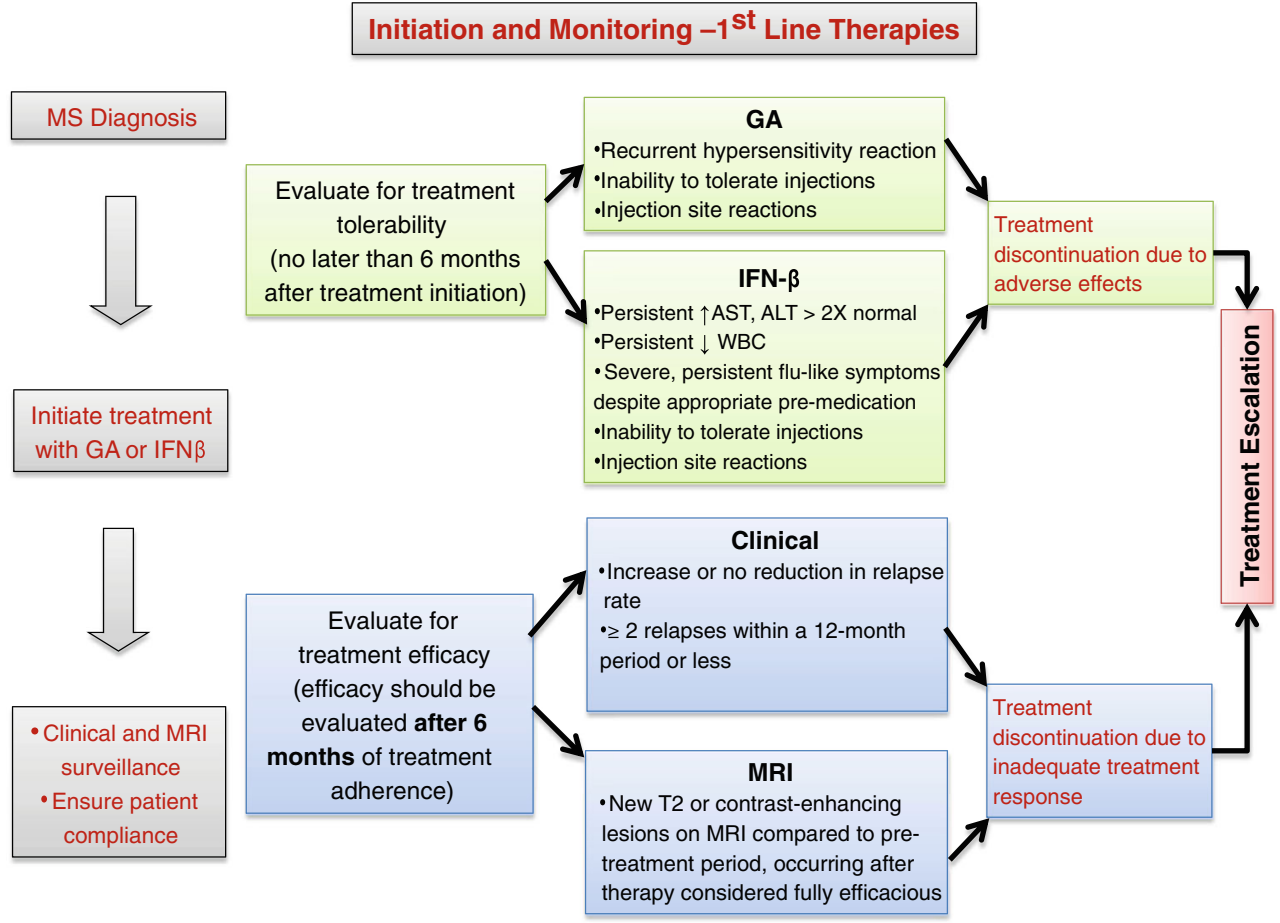

By blocking this integrin's interaction with the vascular endothelium, natalizumab reduces trafficking of immune cells from the periphery into the CNS [80]. Natalizumab is administered as a once-monthly infusion and has demonstrated a high level of efficacy in adults with RRMS; however, the association of natalizumab with progressive multifocal leukoencephalopathy (PML) has limited its use in children and adults. PML is a potentially fatal opportunistic infection of the CNS caused by reactivation of latent John Cunningham (JC) polyomavirus, occurring in immune-suppressed patients. With JC virus infection of oligodendrocytes, cellular lysis occurs and results in widespread, multifocal demyelination [81]. A positive anti-JC virus antibody titer, prior use of immunesuppressant medications, and increased duration of treatment with natalizumab increase the risk of PML [82]. PML risk also appears to correlate directly with anti-JC virus antibody titers [83]. Currently, there are no reported cases of PML secondary to natalizumab use in pediatric patients with MS.

In randomized, placebo-controlled trials in adult MS, comparison of treated patients with those who received placebo revealed an ARR reduction of $68 \%$ over 2 years, sustained reduction in disability progression of $42 \%$, reduction of new or enlarging T2-hyperintense lesions by $83 \%$, and reduction in MRI gadolinium-enhancing lesion number by $92 \%$ [84]. A number of cohort studies evaluating the use of natalizumab in pediatric RRMS have demonstrated that natalizumab is well tolerated and appears to reduce ARR effectively, and sustain disability progression, and T2-hyperintense and gadoliniumenhancing lesion accrual [85-91].

\section{Alemtuzumab}

Alemtuzumab, a monoclonal antibody directed against CD52, received European Medicines Agency (EMA) approval in 2013 followed by FDA approval in 2014 as therapy for RRMS patients who have inadequately responded to $\geq 2 \mathrm{MS}$ therapies. A single, 5-day pulse of this treatment rapidly depletes mature, circulating B and T lymphocytes. This depletion is followed by gradual repopulation of these cells over many months, with CD4+ T lymphocytes recovering last. Phase 3 studies in adult RRMS have shown reduction in relapse rates and sustained disability when compared with IFN- $\beta 1$ a $[92,93]$. The side effect profile is noteworthy and includes infusion reactions, infection, secondary malignancies, and autoimmune disorders (thyroid disease in up to $34 \%$ of patients, nephropathies in $0.3 \%$, and immune thrombocytopenia in $1 \%$ [ [94]. The safety of this therapy in pediatric MS has not been evaluated.

\section{Cyclophosphamide}

Cyclophosphamide is a synthetic anti-neoplastic alkylating agent that interferes with DNA transcription of actively dividing cells. Cyclophosphamide is typically infused monthly (with or without induction) and works as an immunosuppressing agent affecting cytokine expression (with pronounced effects on IL-12) along with T-cell and B-cell function [95]. In adults with RRMS, cyclophosphamide appears to reduce relapse rate and the accrual of new lesions on MRI [96, 97]. A single, multicenter retrospective study of 17 cyclophosphamide- 
treated pediatric patients with MS demonstrated improvements in relapse-related neurologic deficits in the acute setting, in addition to decreased relapse rates and stabilization of disability scores 1 year after treatment initiation [98]. The side effect profile of this therapy limits its use in children and includes nausea/vomiting, alopecia, amenorrhea, osteoporosis, hemorrhagic cystitis, and the risk of secondary malignancy and infertility in both males and females.

\section{Mitoxantrone}

Mitoxantrone is approved for the treatment of worsening adult RRMS; however, given the risk of cardiotoxicity and high rates of leukemia [99], use of this therapy in pediatric MS is discouraged.

\section{Rituximab}

It is now accepted that autoimmune B cells play key roles in establishing MS pathogenesis [100]. A rising interest in the role of humoral immunity occurred when rituximab, a B-celldepleting drug, was shown to reduce relapse rates and the number of gadolinium-enhancing lesions effectively in patients with RRMS [101]. Rituximab is a chimeric monoclonal antibody against CD20, a protein on the surface of pre-B cells and mature B cells. Two retrospective studies assessing the use of rituximab in children with various neuroinflammatory diseases (including 6 pediatric RRMS cases) showed mixed benefits, with only 3 of the 6 patients with RRMS demonstrating evidence of definite clinical improvement. Infusionrelated reactions occurred in $12.5 \%$ [102, 103]. Given these small numbers, more data are needed to determine its utility in the treatment of pediatric MS.

\section{Ocrelizumab}

Similar to rituximab, ocrelizumab is a monoclonal antibody directed against CD20; however, while structurally similar to rituximab, this antibody is more humanized than chimeric. A single phase 2 study in adults with RRMS showed significant reductions in gadolinium-enhancing lesions and relapse rates compared with placebo [104]. Phase 3 adult studies are currently underway.

\section{Oral Therapies}

\section{Fingolimod}

Fingolimod, the first oral drug to receive FDA approval in North America and Europe for adults with RRMS, is a sphingosine-1-phosphate receptor modulator that prevents Tcell egress from peripheral lymphoid tissue into the peripheral and central circulation [105]. Phase 3 studies have shown that fingolimod significantly reduced ARR and MRI activity when compared with placebo and IFN- $\beta 1 \mathrm{a}[106,107]$. Safety issues have been identified, including first-dose bradycardia, the risk of herpes virus dissemination, and macular edema. Three cases of PML in adult patients with RRMS treated with fingolimod (not previously treated with natalizumab) have been reported. The safety in children is unknown, and the effect of fingolimod in patients who are young with active thymic maturation is of concern. A prospective, double-blind, randomized, active-controlled study has begun to evaluate the efficacy and safety of fingolimod versus IFN- $\beta 1$ a (30 $\mu \mathrm{g}$ once weekly) in pediatric patients with MS (PARADIGMS), with a primary endpoint of annualized relapse rate reduction.

\section{Dimethyl Fumarate}

Dimethyl fumarate is an oral fumaric acid ester that also recently gained FDA approval for the treatment of RRMS in adults. Dimethyl fumarate is thought to work by activating nuclear factor erythroid 2-Y-related factor 2, which thereby upregulates antioxidative pathways and reduces expression of inflammatory cytokines, chemokines, and adhesion molecules [108]. Two phase 3 studies have shown that dimethyl fumarate is effective at significantly reducing relapse rate and the number of new or enlarging T2-hyperintense lesions [109, 110]. Though the safety profile was initially thought to be favorable, other than flushing and gastrointestinal disturbance, reports of PML in the setting of dimethyl fumarate have been released [111, 112]. An open-label, randomized, activecontrolled study of dimethyl fumarate versus IFN- $\beta 1$ a (30 $\mu \mathrm{g}$ once weekly) in pediatric patients with MS (CONNECT) is currently recruiting. This study's primary endpoint is the proportion of subjects free of new or newly enlarging T2-hyperintense lesions on brain MRI. A randomized, placebo-controlled trial evaluating the efficacy and safety of dimethyl fumarate (IMAGINE) is in development. In this trial, time to first relapse will serve as the primary endpoint. Finally, an open-label study evaluating the effect of dimethyl fumarate on brain MRI lesions in pediatric patients with MS (FOCUS) is actively enrolling.

\section{Teriflunomide}

Teriflunomide, an FDA-approved oral therapy for adults with RRMS, reversibly inhibits a key mitochondrial enzyme that is involved in pyrimidine synthesis, required for DNA replication. It is thought that this drug preferentially diminishes activation and proliferation of $\mathrm{T}$ - and B-cells (fast-replicating cells) while sparing the proliferation of slow-dividing cells [113]. Studies have shown that teriflunomide significantly reduces relapse rate, disability progression (at higher dose), and MRI evidence of disease activity compared with placebo [114-116]. When looking at risk of treatment failure, 
teriflunomide was not statistically superior to IFN- $\beta 1$ a [117]. Hair thinning and gastrointestinal upset are potential side effects in addition to a black box warning, given the potential for hepatotoxicity and teratogenicity. A washout with cholestyramine or activated charcoal is used to hasten removal of teriflunomide in the event of side effects or an unplanned pregnancy. A randomized, double-blind, placebo-controlled trial is currently underway to evaluate the safety and efficacy of teriflunomide in pediatric MS (TERIKIDS), with time to first clinical relapse serving as the primary outcome measure.

\section{Conclusions and Future Directions}

The need for prospective, randomized, controlled studies for therapies in pediatric MS is paramount. FDA and EMA regulations mandate the inclusion of a pediatric investigation plan for new therapies in attempts to ensure safe access to the new therapeutic agents for pediatric use. Given the rarity of pediatric MS, international efforts to recruit an adequate number of patients for clinical trials is required. In addition, trial design must consider the number of concurrent trials that can feasibly be launched if target trial enrollment is to be achieved.

Acute management of pediatric ADS is essential to maximize recovery. Consensus definitions for adequate and inadequate disease control in pediatric-onset MS are emerging, and are likely to vary when balancing risks and benefits of different therapies. A rationale and evidence-based strategy for serial therapy selection has yet to be proposed or studied and the cumulative risks of multiple therapeutic exposures remain unknown. The goal of preventing future physical and cognitive disability remains paramount, but long-term observation will be required to determine such outcomes. The IPMSSG (www.ipmssg.org) was formed to help address these priorities.

\section{Compliance with Ethical Standards}

Required Author Forms Disclosure forms provided by the authors are available with the online version of this article.

\section{References}

1. Banwell B, Kennedy J, Sadovnick D, et al. Incidence of acquired demyelination of the CNS in Canadian children. Neurology 2009;72:232-239.

2. Langer-Gould A, Zhang JL, Chung J, Yeung Y, Waubant E, Yao J. Incidence of acquired CNS demyelinating syndromes in a multiethnic cohort of children. Neurology 2011;77:1143-1148.

3. Ketelslegers IA, Catsman-Berrevoets CE, Neuteboom RF, et al. Incidence of acquired demyelinating syndromes of the CNS in
Dutch children: a nationwide study. J Neurol 2012;259:19291935.

4. Reinhardt K, Weiss S, Rosenbauer J, Gartner J, von Kries R. Multiple sclerosis in children and adolescents: incidence and clinical picture - new insights from the nationwide German surveillance (2009-2011). Eur J Neurol 2014;21:654-659.

5. Absoud M, Lim MJ, Chong WK, et al. Paediatric acquired demyelinating syndromes: incidence, clinical and magnetic resonance imaging features. Mult Scler 2013;19:76-86.

6. Banwell B, Bar-Or A, Arnold DL, et al. Clinical, environmental, and genetic determinants of multiple sclerosis in children with acute demyelination: a prospective national cohort study. Lancet Neurol 2011;10:436-445.

7. Tantsis EM, Prelog K, Brilot F, Dale RC. Risk of multiple sclerosis after a first demyelinating syndrome in an Australian Paediatric cohort: clinical, radiological features and application of the McDonald 2010 MRI criteria. Mult Scler 2013;19:1749-1759.

8. Mikaeloff Y, Adamsbaum C, Husson B, et al. MRI prognostic factors for relapse after acute CNS inflammatory demyelination in childhood. Brain 2004;127:1942-1947.

9. Dale RC, Pillai SC. Early relapse risk after a first CNS inflammatory demyelination episode: examining international consensus definitions. Dev Med Child Neurol 2007;49:887-893.

10. Simone IL, Carrara D, Tortorella C, et al. Course and prognosis in early-onset MS: comparison with adult-onset forms. Neurology 2002;59:1922-1928.

11. Gorman MP, Healy BC, Polgar-Turcsanyi M, Chitnis T. Increased relapse rate in pediatric-onset compared with adult-onset multiple sclerosis. Arch Neurol 2009;66:54-59.

12. Benson LA, Healy BC, Gorman MP, et al. Elevated relapse rates in pediatric compared to adult MS persist for at least 6 years. Mult Scler Relat Disord 2014;3:186-193.

13. Krupp LB, Tardieu M, Amato MP, et al. International Pediatric Multiple Sclerosis Study Group criteria for pediatric multiple sclerosis and immune-mediated central nervous system demyelinating disorders: revisions to the 2007 definitions. Mult Scler 2013;19: 1261-1267.

14. Mikaeloff Y, Caridade G, Husson B, Suissa S, Tardieu M, Neuropediatric KIDSEP Study Group of the French Neuropediatric Society. Acute disseminated encephalomyelitis cohort study: prognostic factors for relapse. Eur J Paediatr Neurol 2007;11:90-95.

15. Tenembaum S, Chamoles N, Fejerman N. Acute disseminated encephalomyelitis: a long-term follow-up study of 84 pediatric patients. Neurology 2002;59:1224-1231.

16. Anlar B, Basaran C, Kose G, et al. Acute disseminated encephalomyelitis in children: outcome and prognosis. Neuropediatrics 2003;34:194-199.

17. Dale RC, de Sousa C, Chong WK, Cox TC, Harding B, Neville BG. Acute disseminated encephalomyelitis, multiphasic disseminated encephalomyelitis and multiple sclerosis in children. Brain 2000;123:2407-2422.

18. Hynson JL, Kornberg AJ, Coleman LT, Shield L, Harvey AS, Kean MJ. Clinical and neuroradiologic features of acute disseminated encephalomyelitis in children. Neurology 2001;56:13081312 .

19. Kleiman M, Brunquell P. Acute disseminated encephalomyelitis: response to intravenous immunoglobulin. J Child Neurol 1995; 10: 481-483.

20. Atzori M, Battistella PA, Perini P, et al. Clinical and diagnostic aspects of multiple sclerosis and acute monophasic encephalomyelitis in pediatric patients: a single centre prospective study. Mult Scler 2009;15:363-370.

21. Okumura A, Nakazawa M, Igarashi A, et al. Anti-aquaporin 4 antibody-positive acute disseminated encephalomyelitis. Brain Dev 2015;37:339-343. 
22. Baumann M, Sahin K, Lechner C, et al. Clinical and neuroradiological differences of paediatric acute disseminating encephalomyelitis with and without antibodies to the myelin oligodendrocyte glycoprotein. J Neurol Neurosurg Psychiatry 2015;86:265-272.

23. Callen DJ, Shroff MM, Branson HM, et al. Role of MRI in the differentiation of ADEM from MS in children. Neurology 2009;72:968-973.

24. Verhey LH, Branson HM, Shroff MM, et al. MRI parameters for prediction of multiple sclerosis diagnosis in children with acute CNS demyelination: a prospective national cohort study. Lancet Neurol 2011;10:1065-1073.

25. Mikaeloff Y, Suissa S, Vallee L, et al. First episode of acute CNS inflammatory demyelination in childhood: prognostic factors for multiple sclerosis and disability. J Pediatr 2004;144:246-252.

26. Neuteboom RF, Boon M, Catsman Berrevoets CE, et al. Prognostic factors after a first attack of inflammatory CNS demyelination in children. Neurology 2008;71:967-973.

27. Huppke P, Rostasy K, Karenfort M, et al. Acute disseminated encephalomyelitis followed by recurrent or monophasic optic neuritis in pediatric patients. Mult Scler 2013;19:941-946.

28. Titulaer MJ, Hoftberger R, lizuka T, et al. Overlapping demyelinating syndromes and anti-N-methyl-D-aspartate receptor encephalitis. Ann Neurol 2014;75:411-428.

29. Polman CH, Reingold SC, Banwell B, et al. Diagnostic criteria for multiple sclerosis: 2010 revisions to the McDonald criteria. Ann Neurol 2011;69:292-302.

30. Sadaka Y, Verhey LH, Shroff MM, et al. 2010 McDonald criteria for diagnosing pediatric multiple sclerosis. Ann Neurol 2012;72: 211-223.

31. Ghassemi R, Narayanan S, Banwell B, et al. Quantitative determination of regional lesion volume and distribution in children and adults with relapsing-remitting multiple sclerosis. PLoS One 2014;9:e85741

32. Yeh EA, Weinstock-Guttman B, Ramanathan M, et al. Magnetic resonance imaging characteristics of children and adults with paediatric-onset multiple sclerosis. Brain 2009;132:3392-3400.

33. Ghassemi R, Antel SB, Narayanan S, et al. Lesion distribution in children with clinically isolated syndromes. Ann Neurol 2008;63: 401-405.

34. Verhey LH, Signori A, Arnold DL, et al. Clinical and MRI activity as determinants of sample size for pediatric multiple sclerosis trials. Neurology 2013;81:1215-1221.

35. Renoux C, Vukusic S, Mikaeloff Y, et al. Natural history of multiple sclerosis with childhood onset. N Engl J Med 2007;356: 2603-2613.

36. Banwell BL, Anderson PE. The cognitive burden of multiple sclerosis in children. Neurology 2005;64:891-894.

37. Amato MP, Goretti B, Ghezzi A, et al. Cognitive and psychosocial features of childhood and juvenile MS. Neurology 2008;70:1891-1897.

38. Julian L, Serafin D, Charvet L, et al. Cognitive impairment occurs in children and adolescents with multiple sclerosis: results from a United States network. J Child Neurol 2013;28:102-107.

39. Amato MP, Goretti B, Ghezzi A, et al. Neuropsychological features in childhood and juvenile multiple sclerosis: five-year follow-up. Neurology 2014;83:1432-1438.

40. Chitnis T, Tenembaum S, Banwell B, et al. Consensus statement: evaluation of new and existing therapeutics for pediatric multiple sclerosis. Mult Scler 2012;18:116-127.

41. Filippini G, Brusaferri F, Sibley WA, et al. Corticosteroids or ACTH for acute exacerbations in multiple sclerosis. Cochrane Database Syst Rev 2000;(4):CD001331.

42. Brusaferri F, Candelise L. Steroids for multiple sclerosis and optic neuritis: a meta-analysis of randomized controlled clinical trials. J Neurol 2000;247:435-442.
43. Barkhof F, Hommes OR, Scheltens P, Valk J. Quantitative MRI changes in gadolinium-DTPA enhancement after high-dose intravenous methylprednisolone in multiple sclerosis. Neurology 1991;41:1219-1222.

44. Beck RW, Cleary PA, Anderson MM, Jr, et al. A randomized, controlled trial of corticosteroids in the treatment of acute optic neuritis. The Optic Neuritis Study Group. N Engl J Med 1992;326:581-588.

45. Sloka JS, Stefanelli M. The mechanism of action of methylprednisolone in the treatment of multiple sclerosis. Mult Scler 2005;11: 425-432.

46. Martinelli V, Rocca MA, Annovazzi P, et al. A short-term randomized MRI study of high-dose oral vs intravenous methylprednisolone in MS. Neurology 2009;73:1842-1848.

47. Metz LM, Sabuda D, Hilsden RJ, Enns R, Meddings JB. Gastric tolerance of high-dose pulse oral prednisone in multiple sclerosis. Neurology 1999;53:2093-2096.

48. Ramo-Tello C, Grau-Lopez L, Tintore M, et al. A randomized clinical trial of oral versus intravenous methylprednisolone for relapse of MS. Mult Scler 2014;20:717-725.

49. Sahlas DJ, Miller SP, Guerin M, Veilleux M, Francis G. Treatment of acute disseminated encephalomyelitis with intravenous immunoglobulin. Neurology 2000;54:1370-1372.

50. Nishikawa M, Ichiyama T, Hayashi T, Ouchi K, Furukawa S. Intravenous immunoglobulin therapy in acute disseminated encephalomyelitis. Pediatr Neurol 1999;21:583-586.

51. Spalice A, Properzi E, Lo Faro V, Acampora B, Iannetti P. Intravenous immunoglobulin and interferon: successful treatment of optic neuritis in pediatric multiple sclerosis. J Child Neurol 2004; 19:623-626.

52. Elsone L, Panicker J, Mutch K, Boggild M, Appleton R, Jacob A. Role of intravenous immunoglobulin in the treatment of acute relapses of neuromyelitis optica: experience in 10 patients. Mult Scler 2014;20:501-504.

53. Hahn JS, Siegler DJ, Enzmann D. Intravenous gammaglobulin therapy in recurrent acute disseminated encephalomyelitis. Neurology 1996;46:1173-1174.

54. Andersson UG, Bjork L, Skansen-Saphir U, Andersson JP. Downregulation of cytokine production and interleukin-2 receptor expression by pooled human IgG. Immunology 1993;79:211-216.

55. Rossi F, Dietrich G, Kazatchkine MD. Anti-idiotypes against autoantibodies in normal immunoglobulins: evidence for network regulation of human autoimmune responses. Immunol Rev 1989;110:135-149.

56. Weinshenker BG, O'Brien PC, Petterson TM, et al. A randomized trial of plasma exchange in acute central nervous system inflammatory demyelinating disease. Ann Neurol 1999;46:878-886.

57. Rodriguez M, Karnes WE, Bartleson JD, Pineda AA. Plasmapheresis in acute episodes of fulminant CNS inflammatory demyelination. Neurology 1993;43:1100-1104.

58. Mowry EM, Krupp LB, Milazzo M, et al. Vitamin D status is associated with relapse rate in pediatric-onset multiple sclerosis. Ann Neurol 2010;67:618-624.

59. Mikaeloff Y, Caridade G, Tardieu M, Suissa S, KIDSEP study group. Parental smoking at home and the risk of childhood-onset multiple sclerosis in children. Brain 2007;130:2589-2595.

60. Hedstrom AK, Olsson T, Alfredsson L. High body mass index before age 20 is associated with increased risk for multiple sclerosis in both men and women. Mult Scler 2012;18:1334-1336.

61. Langer-Gould A, Brara SM, Beaber BE, Koebnick C. Childhood obesity and risk of pediatric multiple sclerosis and clinically isolated syndrome. Neurology 2013;80:548-552.

62. Bevan CJ, Cree BA. Disease activity free status: a new end point for a new era in multiple sclerosis clinical research? JAMA Neurol 2014;71:269-270. 
63. Thannhauser JE, Mah JK, Metz LM. Adherence of adolescents to multiple sclerosis disease-modifying therapy. Pediatr Neurol 2009;41:119-123.

64. Lulu S, Julian L, Shapiro E, Hudson K, Waubant E. Treatment adherence and transitioning youth in pediatric multiple sclerosis. Mult Scler Relat Disord 2014;3:689-695.

65. Johnson KP, Brooks BR, Cohen JA, et al. Copolymer 1 reduces relapse rate and improves disability in relapsing-remitting multiple sclerosis: results of a phase III multicenter, double-blind, placebocontrolled trial. 1995. Neurology 2001;57(12 Suppl. 5):S16-S24.

66. Farina C, Weber MS, Meinl E, Wekerle H, Hohlfeld R. Glatiramer acetate in multiple sclerosis: update on potential mechanisms of action. Lancet Neurol 2005;4:567-575.

67. Lalive PH, Neuhaus $\mathrm{O}$, Benkhoucha $\mathrm{M}$, et al. Glatiramer acetate in the treatment of multiple sclerosis: emerging concepts regarding its mechanism of action. CNS Drugs 2011;25:401-414.

68. Ghezzi A, Amato MP, Capobianco M, et al. Disease-modifying drugs in childhood-juvenile multiple sclerosis: results of an Italian co-operative study. Mult Scler 2005;11:420-424.

69. Ghezzi A, Immunomodulatory Treatment of Early Onset MS (ITEMS) Group. Immunomodulatory treatment of early onset multiple sclerosis: results of an Italian Co-operative Study. Neurol Sci 2005;26(Suppl. 4):S183-S186.

70. Kornek B, Bernert G, Balassy C, Geldner J, Prayer D, Feucht M. Glatiramer acetate treatment in patients with childhood and juvenile onset multiple sclerosis. Neuropediatrics 2003;34:120-126.

71. Lu E, Wang BW, Guimond C, Synnes A, Sadovnick D, Tremlett $\mathrm{H}$. Disease-modifying drugs for multiple sclerosis in pregnancy: a systematic review. Neurology 2012;79:1130-1135.

72. Kieseier BC. The mechanism of action of interferon-beta in relapsing multiple sclerosis. CNS Drugs 2011;25:491-502.

73. Mikaeloff Y, Moreau T, Debouverie M, et al. Interferon-beta treatment in patients with childhood-onset multiple sclerosis. J Pediatr 2001;139:443-446.

74. Pohl D, Rostasy K, Gartner J, Hanefeld F. Treatment of early onset multiple sclerosis with subcutaneous interferon beta-1a. Neurology 2005;64:888-890.

75. Tenembaum SN, Segura MJ. Interferon beta-1 a treatment in childhood and juvenile-onset multiple sclerosis. Neurology 2006;67: 511-513.

76. Banwell B, Reder AT, Krupp L, et al. Safety and tolerability of interferon beta-1b in pediatric multiple sclerosis. Neurology 2006;66:472-476.

77. Mikaeloff Y, Caridade G, Tardieu M, Suissa S, KIDSEP study group of the French Neuropediatric Society. Effectiveness of early beta interferon on the first attack after confirmed multiple sclerosis: a comparative cohort study. Eur J Paediatr Neurol 2008;12: 205-209.

78. Ghezzi A, Banwell B, Boyko A, et al. The management of multiple sclerosis in children: a European view. Mult Scler 2010;16: 1258-1267.

79. Yeh EA, Waubant E, Krupp LB, et al. Multiple sclerosis therapies in pediatric patients with refractory multiple sclerosis. Arch Neurol 2011;68:437-444.

80. Rudick RA, Sandrock A. Natalizumab: alpha 4-integrin antagonist selective adhesion molecule inhibitors for MS. Expert Rev Neurother 2004;4:571-580.

81. Aksamit AJ, Jr. Progressive multifocal leukoencephalopathy: a review of the pathology and pathogenesis. Microsc Res Tech 1995;32:302-311.

82. Bloomgren $\mathrm{G}$, Richman $\mathrm{S}$, Hotermans $\mathrm{C}$, et al. Risk of natalizumabassociated progressive multifocal leukoencephalopathy. N Engl J Med 2012;366:1870-1880.

83. Plavina T, Subramanyam M, Bloomgren G, et al. Anti-JC virus antibody levels in serum or plasma further define risk of natalizumab-associated progressive multifocal leukoencephalopathy. Ann Neurol 2014;76:802-812.

84. Polman $\mathrm{CH}$, O'Connor PW, Havrdova E, et al. A randomized, placebo-controlled trial of natalizumab for relapsing multiple sclerosis. N Engl J Med 2006;354:899-910.

85. Ghezzi A, Pozzilli C, Grimaldi LM, et al. Natalizumab in pediatric multiple sclerosis: results of a cohort of 55 cases. Mult Scler 2013;19:1106-1112.

86. Ghezzi A, Pozzilli C, Grimaldi LM, et al. Safety and efficacy of natalizumab in children with multiple sclerosis. Neurology 2010;75:912-917.

87. Arnal-Garcia C, Garcia-Montero MR, Malaga I, et al. Natalizumab use in pediatric patients with relapsing-remitting multiple sclerosis. Eur J Paediatr Neurol 2013;17:50-54.

88. Kornek B, Aboul-Enein F, Rostasy K, et al. Natalizumab therapy for highly active pediatric multiple sclerosis. JAMA Neurol 2013;70:469-475.

89. Yeh EA, Weinstock-Guttman B. Natalizumab in pediatric multiple sclerosis patients. Ther Adv Neurol Disord 2010;3:293-299.

90. Huppke P, Stark W, Zurcher C, Huppke B, Bruck W, Gartner J. Natalizumab use in pediatric multiple sclerosis. Arch Neurol 2008; 65:1655-1658

91. Ghezzi A, Moiola L, Pozzilli C, et al. Natalizumab in the pediatric MS population: results of the Italian registry. BMC Neurol 2015; 15:174-015-0433-y.

92. Cohen JA, Coles AJ, Arnold DL, et al. Alemtuzumab versus interferon beta 1a as first-line treatment for patients with relapsingremitting multiple sclerosis: a randomised controlled phase 3 trial. Lancet 2012;380:1819-1828.

93. Coles AJ, Twyman CL, Arnold DL, et al. Alemtuzumab for patients with relapsing multiple sclerosis after disease-modifying therapy: a randomised controlled phase 3 trial. Lancet 2012;380: 1829-1839.

94. Hartung HP, Aktas O, Boyko AN. Alemtuzumab: a new therapy for active relapsing-remitting multiple sclerosis. Mult Scler 2015;21:22-34.

95. Weiner HL, Cohen JA. Treatment of multiple sclerosis with cyclophosphamide: critical review of clinical and immunologic effects. Mult Scler 2002;8:142-154.

96. Smith DR, Weinstock-Guttman B, Cohen JA, et al. A randomized blinded trial of combination therapy with cyclophosphamide in patients-with active multiple sclerosis on interferon beta. Mult Scler 2005;11:573-582.

97. Reggio E, Nicoletti A, Fiorilla T, Politi G, Reggio A, Patti F. The combination of cyclophosphamide plus interferon beta as rescue therapy could be used to treat relapsing-remitting multiple sclerosis patients - twenty-four months follow-up. J Neurol 2005;252: 1255-1261.

98. Makhani N, Gorman MP, Branson HM, Stazzone L, Banwell BL, Chitnis T. Cyclophosphamide therapy in pediatric multiple sclerosis. Neurology 2009;72:2076-2082.

99. Marriott JJ, Miyasaki JM, Gronseth G, O'Connor PW, Therapeutics and Technology Assessment Subcommittee of the American Academy of Neurology. Evidence Report: The efficacy and safety of mitoxantrone (Novantrone) in the treatment of multiple sclerosis: Report of the Therapeutics and Technology Assessment Subcommittee of the American Academy of Neurology. Neurology 2010;74:1463-1470.

100. Owens GP, Bennett JL, Gilden DH, Burgoon MP. The B cell response in multiple sclerosis. Neurol Res 2006;28:236-244.

101. Hauser SL, Waubant E, Arnold DL, et al. B-cell depletion with rituximab in relapsing-remitting multiple sclerosis. N Engl J Med 2008;358:676-688.

102. Dale RC, Brilot F, Duffy LV, et al. Utility and safety of rituximab in pediatric autoimmune and inflammatory CNS disease. Neurology 2014;83:142-150. 
103. Beres SJ, Graves J, Waubant E. Rituximab use in pediatric central demyelinating disease. Pediatr Neurol 2014;51:114-118.

104. Kappos L, Li D, Calabresi PA, et al. Ocrelizumab in relapsingremitting multiple sclerosis: a phase 2, randomised, placebocontrolled, multicentre trial. Lancet 2011;378:1779-1787.

105. Pelletier D, Hafler DA. Fingolimod for multiple sclerosis. N Eng1 J Med 2012;366:339-347.

106. Kappos L, Radue EW, O'Connor P, et al. A placebo-controlled trial of oral fingolimod in relapsing multiple sclerosis. N Engl J Med 2010;362:387-401.

107. Cohen JA, Barkhof F, Comi G, et al. Oral fingolimod or intramuscular interferon for relapsing multiple sclerosis. N Engl J Med 2010;362:402-415.

108. Gold R, Linker RA, Stangel M. Fumaric acid and its esters: an emerging treatment for multiple sclerosis with antioxidative mechanism of action. Clin Immunol 2012;142: 44-48.

109. Gold R, Kappos L, Arnold DL, et al. Placebo-controlled phase 3 study of oral BG-12 for relapsing multiple sclerosis. N Engl J Med 2012;367:1098-1107.

110. Fox RJ, Miller DH, Phillips JT, et al. Placebo-controlled phase 3 study of oral BG-12 or glatiramer in multiple sclerosis. N Engl J Med 2012;367:1087-1097.
111. Nieuwkamp DJ, Murk JL, van Oosten BW, et al. PML in a patient without severe lymphocytopenia receiving dimethyl fumarate. N Engl J Med 2015;372:1474-1476.

112. Rosenkranz T, Novas M, Terborg C. PML in a patient with lymphocytopenia treated with dimethyl fumarate. N Engl J Med 2015;372:1476-1478.

113. Fox RI, Herrmann ML, Frangou CG, et al. Mechanism of action for leflunomide in rheumatoid arthritis. Clin Immunol 1999;93:198-208.

114. O'Connor P, Wolinsky JS, Confavreux C, et al. Randomized trial of oral teriflunomide for relapsing multiple sclerosis. N Engl J Med 2011;365:1293-1303.

115. Miller AE, O'Connor P, Wolinsky JS, et al. Pre-specified subgroup analyses of a placebo-controlled phase III trial (TEMSO) of oral teriflunomide in relapsing multiple sclerosis. Mult Scler 2012;18: 1625-1632.

116. Confavreux $\mathrm{C}, \mathrm{O}^{\prime}$ Connor $\mathrm{P}, \mathrm{Comi} \mathrm{G}$, et al. Oral teriflunomide for patients with relapsing multiple sclerosis (TOWER): a randomised, double-blind, placebo-controlled, phase 3 trial. Lancet Neurol 2014;13:247-256.

117. Vermersch P, Czlonkowska A, Grimaldi LM, et al. Teriflunomide versus subcutaneous interferon beta- $1 \mathrm{a}$ in patients with relapsing multiple sclerosis: a randomised, controlled phase 3 trial. Mult Scler 2014;20:705-716. 